\title{
Polyethyleneimine-functionalized boron nitride nanospheres as efficient carriers for enhancing the immunostimulatory effect of CPG oligodeoxynucleotides
}

\author{
This article was published in the following Dove Press journal: \\ International Journal of Nanomedicine \\ 24 August 2015 \\ Number of times this article has been viewed
}

\author{
Huijie Zhang' \\ Shini Feng' \\ Ting Yan' \\ Chunyi Zhi \\ Xiao-Dong Gao' \\ Nobutaka Hanagata ${ }^{3,4}$ \\ 'The Key Laboratory of Carbohydrate \\ Chemistry and Biotechnology, \\ Ministry of Education, School of \\ Biotechnology, Jiangnan University, \\ Wuxi, People's Republic of China; \\ ${ }^{2}$ Department of Physics and Materials \\ Science, City University of Hong Kong, \\ Kowlong, Hong Kong SAR, People's \\ Republic of China; ${ }^{3}$ Biomaterials Unit, \\ International Center for Materials \\ Nanoarchitectonics, National Institute \\ for Materials Science, Ibaraki, Japan; \\ ${ }^{4}$ Nanotechnology Innovation Station, \\ National Institute for Materials \\ Science, Ibaraki, Japan
}

Correspondence: Huijie Zhang The Key Laboratory of Carbohydrate Chemistry and Biotechnology, Ministry of Education, School of Biotechnology, Jiangnan University, No 1800 Lihu Avenue, Wuxi 214I22, People's Republic of China

$\mathrm{Tel}+8651085197071$

Fax +86 510 8519 7071

Email zhj0502@jiangnan.edu.cn

Nobutaka Hanagata

Nanotechnology Innovation Station,

National Institute for Materials Science,

I-2-I Sengen, Tsukuba, Ibaraki 305-0047, Japan

Tel +8I 298604774

Fax +8I 298592475

Email hanagata.nobutaka@nims.go.jp

\begin{abstract}
CpG}$ oligodeoxynucleotides (ODNs) stimulate innate and adaptive immune responses. Thus, these molecules are promising therapeutic agents and vaccine adjuvants against various diseases. In this study, we developed a novel CpG ODNs delivery system based on polyethyleneimine (PEI)-functionalized boron nitride nanospheres (BNNS). PEI was coated on the surface of BNNS via electrostatic interactions. The prepared BNNS-PEI complexes had positive zeta potential and exhibited enhanced dispersity and stability in aqueous solution. In vitro cytotoxicity assays revealed that the BNNS-PEI complexes with concentrations up to $100 \mu \mathrm{g} / \mathrm{mL}$ exhibited no obvious cytotoxicity. Furthermore, the positively charged surface of the BNNSPEI complexes greatly improved the loading capacity and cellular uptake efficiency of $\mathrm{CpG}$ ODNs. Class B CpG ODNs loaded on the BNNS-PEI complexes enhanced the production of interleukin- 6 and tumor necrosis factor- $\alpha$ from peripheral blood mononuclear cells compared with CpG ODNs directly loaded on BNNS. Contrary to the free CpG ODNs or CpG ODNs directly loaded on BNNS, class B CpG ODNs loaded on the BNNS-PEI complexes induced interferon- $\alpha$ simultaneously. PEI coating may have changed the physical form of class B CpG ODNs on BNNS, which further affected their interaction with Toll-like receptor 9 and induced interferon- $\alpha$. Therefore, BNNS-PEI complexes can be used to enhance the immunostimulatory effect and therapeutic activity of $\mathrm{CpG}$ ODNs and the treatment of diseases requiring interleukin-6, tumor necrosis factor- $\alpha$, and interferon- $\alpha$.
\end{abstract}

Keywords: $\mathrm{CpG}$ oligodeoxynucleotide, boron nitride nanosphere, polyethyleneimine, immunostimulatory effect, cytokine

\section{Introduction}

Bacterial DNA exerts immunostimulatory effects by activating the host defense system and inducing innate and adaptive immune responses. ${ }^{1-4}$ Unmethylated $\mathrm{CpG}$ motif is the specific sequence present in bacterial DNA that is responsible for the aforementioned effects. ${ }^{1,5}$ Similar to bacterial DNA, synthetic oligodeoxynucleotides (ODNs) containing unmethylated $\mathrm{CpG}$ motifs exhibit immunostimulatory effects. ${ }^{1,6} \mathrm{CpG}$ ODNs uptaken by immune cells bind to intracellular Toll-like receptor 9 (TLR-9), a patternrecognition receptor located mainly in the endolysosomes of B cells and plasmacytoid dendritic cells. ${ }^{2,3,7}$ The binding process initiates an immunostimulatory cascade that induces the maturation, differentiation, and proliferation of multiple immune cells, such as B and T lymphocytes, natural killer cells, and monocytes/macrophages. ${ }^{8,9}$ This mechanism further triggers cell signaling pathways, such as mitogen-activated protein kinases and $\mathrm{NF} \kappa \mathrm{B}$, which induce multiple proinflammatory cytokines and chemokines 
as well as modulate cellular inflammatory response. ${ }^{10,11}$ Thus, $\mathrm{CpG}$ ODNs are promising immune adjuvants and immunotherapeutic agents against allergy/asthma, cancer, and infectious diseases. ${ }^{11-14}$ Clinical studies suggest that CpG ODNs are safe and well tolerated when administered to humans; these molecules reportedly improve vaccineinduced immune responses. ${ }^{2,15}$ However, the biologic activity of CpG ODNs is transient, and the extreme susceptibility to nuclease degradation in serum and poor cellular uptake of natural $\mathrm{CpG}$ ODNs severely limit the therapeutic applications of these molecules. ${ }^{16,17}$ Therefore, developing approaches to optimize the stimulatory activity of CpG ODNs has attracted considerable interest. Various strategies are involved in the chemical modification of the $\mathrm{CpG}$ ODNs backbone. For instance, substituting oxygen with sulfur generates phosphorothioate $\mathrm{CpG}$ ODNs, which are highly resistant to nuclease degradation and exert strong immunostimulatory effects. ${ }^{17,18}$ However, modification of the CpG ODNs backbone causes several severe side effects. ${ }^{19,20}$ Thus, numerous studies have explored delivery systems that would improve the stimulatory activity of $\mathrm{CpG}$ ODNs. Increasing evidence indicates that both innate and adaptive immune responses induced by $\mathrm{CpG}$ ODNs can be significantly enhanced using various nanoparticles as carriers. ${ }^{17,18}$ The exact mechanisms have not been defined, but several possible routes have been proposed. These mechanisms include the protection of CpG ODNs from nuclease degradation, prolonged half-life, increased cellular uptake efficiency, and enhanced delivery to intracellular compartments. ${ }^{18}$ Therefore, using nanoparticles as carriers may facilitate the use of naturally occurring $\mathrm{CpG}$ ODNs in clinical applications.

Boron nitride $(\mathrm{BN})$ has attracted considerable attention because of its outstanding properties and structural similarity with carbon. ${ }^{21,22} \mathrm{BN}$ also exhibits easier cellular uptake and lower cytotoxicity than carbon materials. ${ }^{23,24}$ Therefore, BN has several applications ranging from composite materials to electrical and optical devices. ${ }^{22,25} \mathrm{BN}$ nanomaterials also have biomedical applications. In vitro and in vivo experiments suggested that BN nanotubes possess optimal biocompatibility, indicating their potential biomedical applications. ${ }^{26-28}$ We previously used boron nitride nanospheres (BNNS) as carriers for the delivery of unmodified $\mathrm{CpG}$ ODNs to activate TLR-9. ${ }^{29}$ BNNS exhibited no cytotoxicity and protected unmodified $\mathrm{CpG}$ ODNs from nuclease degradation. When we added BNNS to 293XL-TLR-9 cells, the BNNS localized in the endolysosome after 24 hours. This localization was maintained even after cell division. TLR-9 also localized in the endolysosome; hence, BNNS can deliver CpG ODNs into the endolysosome compartments of immune cells and stimulate a strong immune response compared with free $\mathrm{CpG}$ ODNs. However, CpG ODNs and BNNS are both negatively charged. Therefore, the loading capacity and cellular uptake efficiency of $\mathrm{CpG}$ ODNs may be limited by electrostatic repulsion and may not induce a robust cytokine response. Furthermore, class B CpG ODNs loaded on BNNS only induced interleukin 6 (IL-6) and tumor necrosis factor- $\alpha$ (TNF- $\alpha$ ) but not interferon- $\alpha$ (IFN- $\alpha$ ). However, a high amount of IFN- $\alpha$ induction can be observed when class B CpG ODNs are adsorbed electrostatically onto polystyrene nanoparticles. ${ }^{30}$ We hypothesized that methods for enhancing CpG ODNs internalization by immune cells may potentiate their immunostimulatory effect. Moreover, CpG ODNs may induce IFN- $\alpha$ when class B CpG ODNs are loaded electrostatically onto BNNS.

In this study, we developed a novel CpG ODNs delivery system based on polyethyleneimine (PEI)-functionalized BNNS (Figure 1). PEI is the "gold standard" cationic polymer for nucleic acid delivery, either used alone or for surface coating of nanoparticles, to bind negatively charged nucleic acid drugs. ${ }^{31,32}$ PEI exhibits high transfection efficiency because of enhanced cellular uptake and endosomal escape. Compared with high-molecular weight PEIs, low-molecular weight PEIs are less toxic and more effective as a coating material for nanoparticles to enhance the immunostimulatory effect of CpG ODNs. ${ }^{33}$ We found that PEI can be coated on negatively charged BNNS through electrostatic interactions, forming BNNS-PEI complexes highly enriched in positive charges that allowed the effective loading of $\mathrm{CpG}$ ODNs via a similar process. The formed BNNS-PEI complexes showed no cytotoxicity and significantly enhanced the loading capacity and cellular uptake of CpG ODNs, further inducing a robust cytokine response. Contrary to free $\mathrm{CpG}$ ODNs or free CpG ODNs directly loaded on BNNS, class B CpG ODNs loaded on the BNNS-PEI complexes can induce IFN- $\alpha$. This phenomenon suggests that coating of PEI on BNNS increased the cytokines stimulated by CpG ODNs possibly by altering signal transduction through the interaction of CpG ODNs with TLR-9. Further investigation on the mechanism underlying this phenomenon is currently underway. The current work may provide a promising strategy to enhance the delivery efficiency and immunostimulatory effect of $\mathrm{CpG}$ ODNs. Class B CpG ODNs loaded on the BNNS-PEI complexes can simultaneously induce IL-6, TNF- $\alpha$, and IFN- $\alpha$. Thus, this study may be beneficial for treating diseases (eg, cancer) requiring the three aforementioned cytokines. 

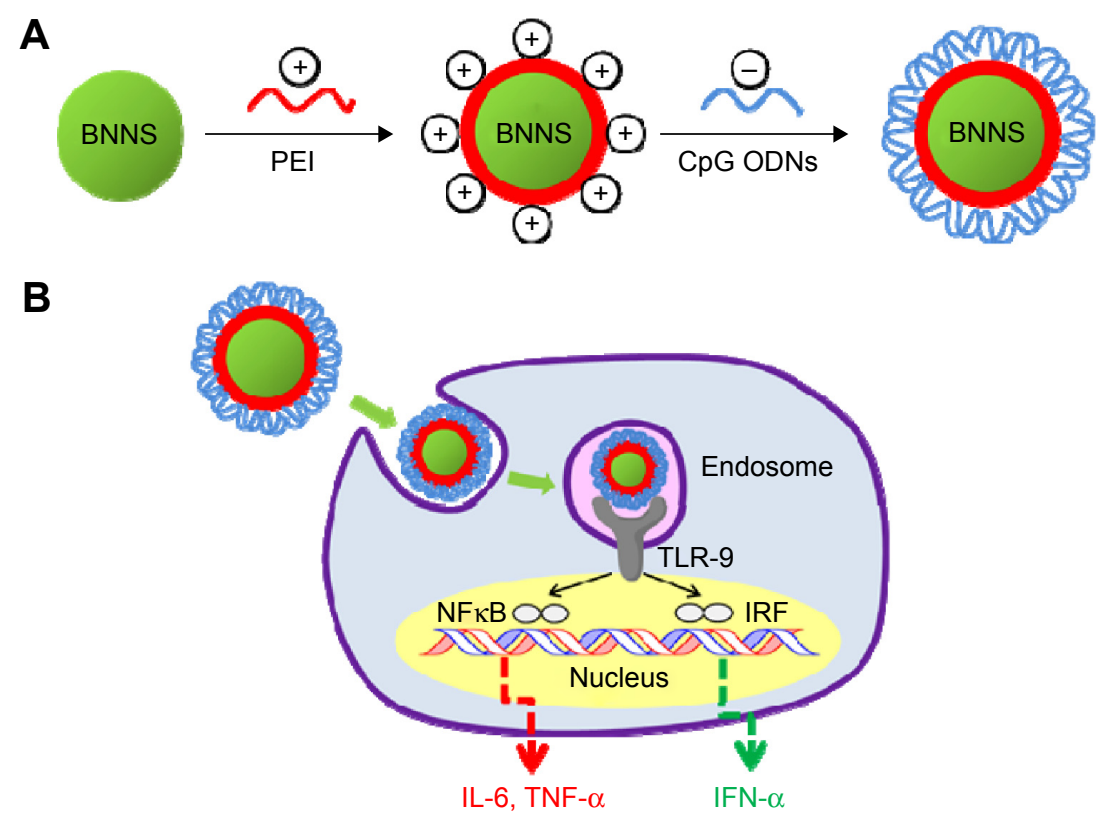

Figure I Schematic illustration of the process for preparation and application of the PEl-functionalized BNNS as an efficient CpG ODNs carrier.

Notes: (A) Preparation of the PEl-functionalized BNNS for CpG ODNs loading. (B) Application of the PEl-functionalized BNNS as a carrier for enhancing the immunostimulatory effects of the CPG ODNs.

Abbreviations: BNNS, boron nitride nanospheres; IFN, interferon; IL-6, interleukin-6; IRF, interferon regulatory factor; NFKB, nuclear factor KB; ODN, oligodeoxynucleotide; PEl, polyethyleneimine; TLR-9, Toll-like receptor 9; TNF, tumor necrosis factor.

\section{Materials and methods Materials}

Branched PEI with an average molecular weight of $600 \mathrm{Da}$ was purchased from Wako Pure Chemicals (Osaka, Japan). Phosphodiester-based class B CpG ODNs, referred to as $\mathrm{CpG}$ ODNs2006x3-PD, ${ }^{34}$ were obtained from Fasmac, Inc. (Kanagawa, Japan), diluted in sterile water at $100 \mu \mathrm{M}$, and then stored at $-20^{\circ} \mathrm{C}$. Frozen human peripheral blood mononuclear cells (PBMCs) were purchased from Cellular Technology Limited (Cleveland, OH, USA) and thawed in accordance with the manufacturer's protocol. Roswell Park Memorial Institute (RPMI)-1640 medium and fetal bovine serum were obtained from Gibco (Grand Island, NY, USA). Cell Counting Kit-8 was purchased from Dojindo (Kumamoto, Japan).

\section{Preparation of BNNS-PEI complexes}

High-purity BNNS were synthesized through chemical vapor deposition as previously reported. ${ }^{35}$ PEI was diluted with Milli-Q water to a concentration of $10 \%(\mathrm{v} / \mathrm{v})$ prior to use. Working solutions were prepared from the stock by dilution with Milli-Q water. BNNS were functionalized by suspending $1 \mathrm{mg}$ of BNNS in $1 \mathrm{~mL}$ of PEI solution, and the resulting solution was stirred at room temperature for 2 hours. The PEI-functionalized BNNS were washed five times with Milli-Q water by centrifugation and redispersed in phosphate-buffered saline (PBS).

\section{Characterizations}

Fourier transform infrared (FTIR) spectroscopy was performed using a Spectrum GX spectrophotometer (Perkin Elmer, Boston, MA, USA) at $4 \mathrm{~cm}^{-1}$ resolution with 32 scans. Transmission electron microscopy was conducted using a $3000 \mathrm{~F}$, high-resolution, field-emission transmission electron microscope (JEOL, Tokyo, Japan) operated at an acceleration voltage of $300 \mathrm{kV}$. Dynamic light scattering measurements were obtained using Photal DLS-6000DL (Otsuka, Hirakata, Japan). Zeta potentials were measured using a LEZA-600 electrophoresis zeta potential analyzer (Otsuka, Japan). UV-vis absorption spectra were measured with a U-2900 spectrophotometer (Hitachi, Tokyo, Japan) at room temperature. Thermogravimetric analysis was conducted on an SII TG/DTA 6200 system at a heating rate of $5^{\circ} \mathrm{C} /$ minute with an open alumina cell.

\section{Cell culture and in vitro cytotoxicity assay}

Frozen human PBMCs were thawed in accordance with the manufacturer's protocol. The cytotoxicities of BNNS and the BNNS-PEI complexes to PBMCs were evaluated using a Cell Counting Kit- 8 assay. PBMCs were seeded into a 96-well plate at a density of $5 \times 10^{4}$ cells per well. After 24 hours of incubation at $37^{\circ} \mathrm{C}$ with $5 \% \mathrm{CO}_{2}$, until the cells adhered to the plate, the cell culture was added with a series of concentrations of BNNS and the BNNS-PEI complexes. 
After another 24 hours of incubation, each well had $10 \mu \mathrm{L}$ of Cell Counting Kit-8 solution and then incubated for another 3 hours. Finally, the absorbance at $450 \mathrm{~nm}$ was obtained using a microplate reader to determine the relative cell viability. No ethics approval was required from the institutional review board for the use of this cell line.

\section{Preparation of BNNS-PEI/CpG ODN complexes}

CpG ODNs solution $(25 \mu \mathrm{L})$ was added to an equal volume of BNNS-PEI solution ( $2 \mathrm{mg} / \mathrm{mL}$ in PBS at $\mathrm{pH} 7.4$ ) and then shaken at room temperature for 1 hour. The mixture was then centrifuged at 15,000 revolutions per minute for 15 minutes to collect the BNNS-PEI/CpG ODN complexes. Binding of the CpG ODNs to the BNNS-PEI complexes was further confirmed through gel electrophoresis using 10\%-20\% gel. BNNS-PEI was incubated with CpG ODNs at various weight ratios (BNNS-PEI/CpG ODNs) from 5 to 100. The mixture was centrifuged, and the supernatant was collected for gel electrophoresis. The CpG ODNs in the gel were visualized by ethidium bromide using a Dolphin-Doc UV transilluminator (Kurabo, Japan). Loading capacity was calculated from the concentration of the unloaded CpG ODNs in the supernatant measured by a NanoDrop 2000 spectrophotometer (Thermo Scientific, Waltham, MA, USA). The BNNS-PEI/CpG ODN complexes were resuspended by adding $50 \mu \mathrm{L}$ of PBS and used for the following cytokine stimulation.

\section{Uptake of CpG ODNs by human PBMCs} Human PBMCs $\left(8 \times 10^{4}\right)$ were seeded in a $35 \mathrm{~mm}$ Petri dish with a glass bottom and then incubated for 24 hours at $37^{\circ} \mathrm{C}$ under $5 \% \mathrm{CO}_{2}$. BNNS/ fluorescein isothiocyanate (FITC)CpG ODN and BNNS-PEI/FITC-CpG ODN complexes were then added to the dish at a final concentration of $50 \mu \mathrm{g} / \mathrm{mL}$. Free FITC-CpG ODNs and BNNS were used as controls. After incubation for 24 hours, the cells were washed twice with cold PBS to terminate the uptake and then fixed with $3.7 \%(\mathrm{v} / \mathrm{v})$ paraformaldehyde. Fluorescence of the fixed cells was visualized under an SP5 confocal laser scanning microscope (Leica, Nussloch, Germany) with a $63 \times$ oil immersion objective.

\section{Cytokine assay}

Human PBMCs were seeded at a density of $5 \times 10^{6}$ cells $/ \mathrm{mL}$ in RPMI-1640 medium supplemented with $10 \%$ fetal bovine serum and then immediately stimulated with the BNNS/CpG ODN and BNNS-PEI/CpG ODN complexes. After 48 hours and 8 hours of incubation at $37^{\circ} \mathrm{C}$ respectively, cell supernatants were collected for further analysis. The concentrations of IL-6, IFN- $\alpha$, and TNF- $\alpha$ in the medium were determined through enzyme-linked immunosorbent assay using the Ready-SET-Go! human IL-6 kit (eBioscience, San Diego, CA, USA), human TNF- $\alpha$ kit (eBioscience), and human IFN-alpha-Module set (eBioscience) in accordance with the manufacturers' protocol.

\section{Statistical analysis}

Statistical analysis was performed using Student's $t$-test. Data are presented as mean \pm standard deviation $(* P<0.05$, $* * P<0.01)$

\section{Results and discussion Synthesis and characterization of the BNNS-PEI complexes}

BNNS were synthesized through chemical vapor deposition. ${ }^{35}$ The as-prepared BNNS showed a uniform spherical shape with an average diameter of approximately $150 \mathrm{~nm}$ (Figure 2A). In addition, well-ordered channels were clearly observed from the high-resolution transmission electron micrograph of the BNNS (Figure 2B). We used PEI to functionalize the surface of BNNS and obtain a positively charged
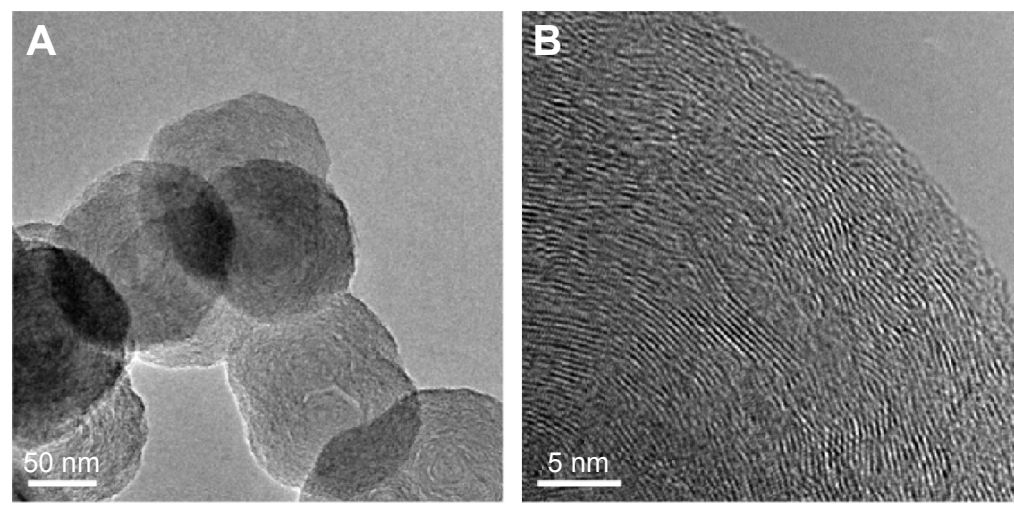

Figure 2 (A) Typical transmission electron microscopy image of the boron nitride nanosphere. (B) A high-resolution image. 
surface that facilitates the loading of $\mathrm{CpG}$ ODNs onto the BNNS. Figure 1 shows the schematic of the synthesis route. BNNS were first noncovalently functionalized by PEI polymers, forming positively charged BNNS-PEI complexes. Then, the negatively charged $\mathrm{CpG}$ ODNs were loaded onto the BNNS-PEI complexes via electrostatic interactions. FTIR results show the PEI coating on BNNS. Figure $3 \mathrm{~A}$ shows the FTIR spectra of BNNS, PEI, and the BNNS-PEI complexes. Two distinct peaks at $2,700-2,900 \mathrm{~cm}^{-1}$ assigned to the $\mathrm{C}-\mathrm{H}$ vibrations in PEI were clearly observed in the BNNS-PEI complexes compared with pure BNNS. This result suggests that PEI has been functionalized on the surface of BNNS. Zeta potential measurements further confirmed the successful functionalization of the BNNS with PEI (Figure 3B). The coating of PEI greatly increased the zeta potential of BNNS from $-5 \mathrm{mV}$ to $+40 \mathrm{mV}$ for the BNNS-PEI complexes. In addition, the surface charge of the BNNS-PEI complexes increased when the initial concentration of the PEI solution was increased during the functionalization of the BNNS. This phenomenon was probably due to the increased amino groups coated on the surface of BNNS. PEI concentrations were optimized to $5 \%$ to achieve the highest positive zeta potential and were used in the following studies. Therefore, the positive charge of the BNNS-PEI complexes facilitated the loading of the negatively charged CpG ODNs through electrostatic interactions and enhanced the cellular uptake of the complexes. Thermogravimetric analysis is a convenient technique that reveals the composition and change in the thermal stability of complexes. This process was used to determine the amount of PEI coated on the surface of BNNS. Figure $3 \mathrm{C}$ shows that BNNS only exhibited slight mass loss $(<5 \%)$ because of their superb structural stability and antioxidation ability. By contrast, a high weight loss (22\%) was observed for the BNNS-PEI complexes. Thus, the weight ratio of PEI in the BNNS-PEI complexes was calculated to be approximately $17 \%$, which possibly facilitated the loading of the CpG ODNs on the BNNS. The dispersity and stability of nanoparticles are crucial for their application as carriers in drug delivery systems. PEI coating enhanced these characteristics of BNNS in physiological solutions (Figure 4A)
A

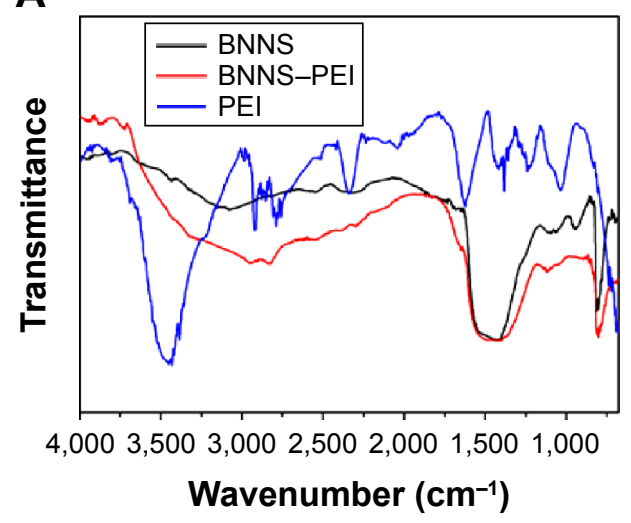

B

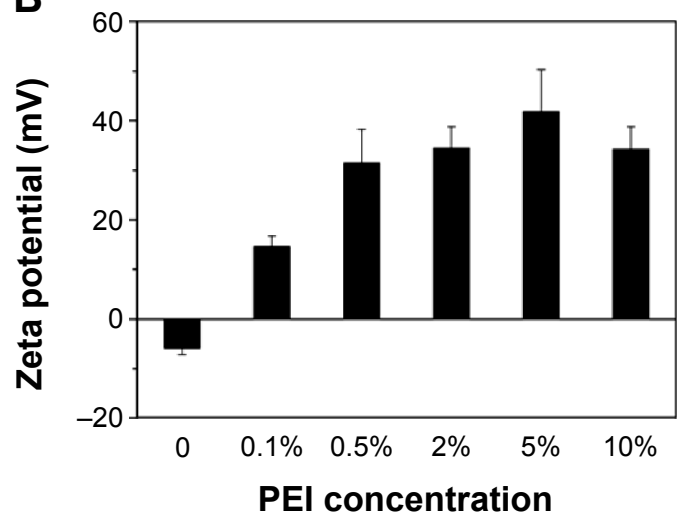

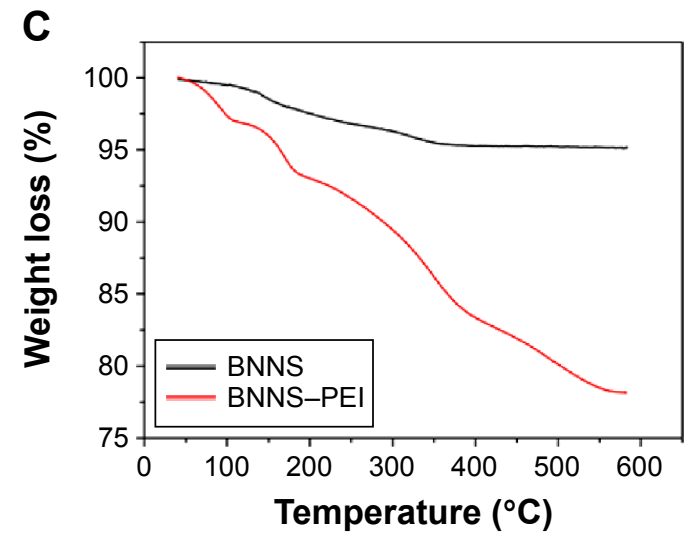

Figure 3 Characterizations of the BNNS and BNNS-PEI complexes.

Notes: (A) FTIR spectra of BNNS, PEI, and BNNS-PEI complexes. (B) Zeta potentials of BNNS and a series of BNNS-PEl complexes at different starting concentrations of PEI. (C) Thermal gravimetric analysis of the BNNS and BNNS-PEI complexes.

Abbreviations: BNNS, boron nitride nanospheres; FTIR, Fourier transform infrared; PEl, polyethyleneimine. 


\section{A BNNS-PEI BNNS}
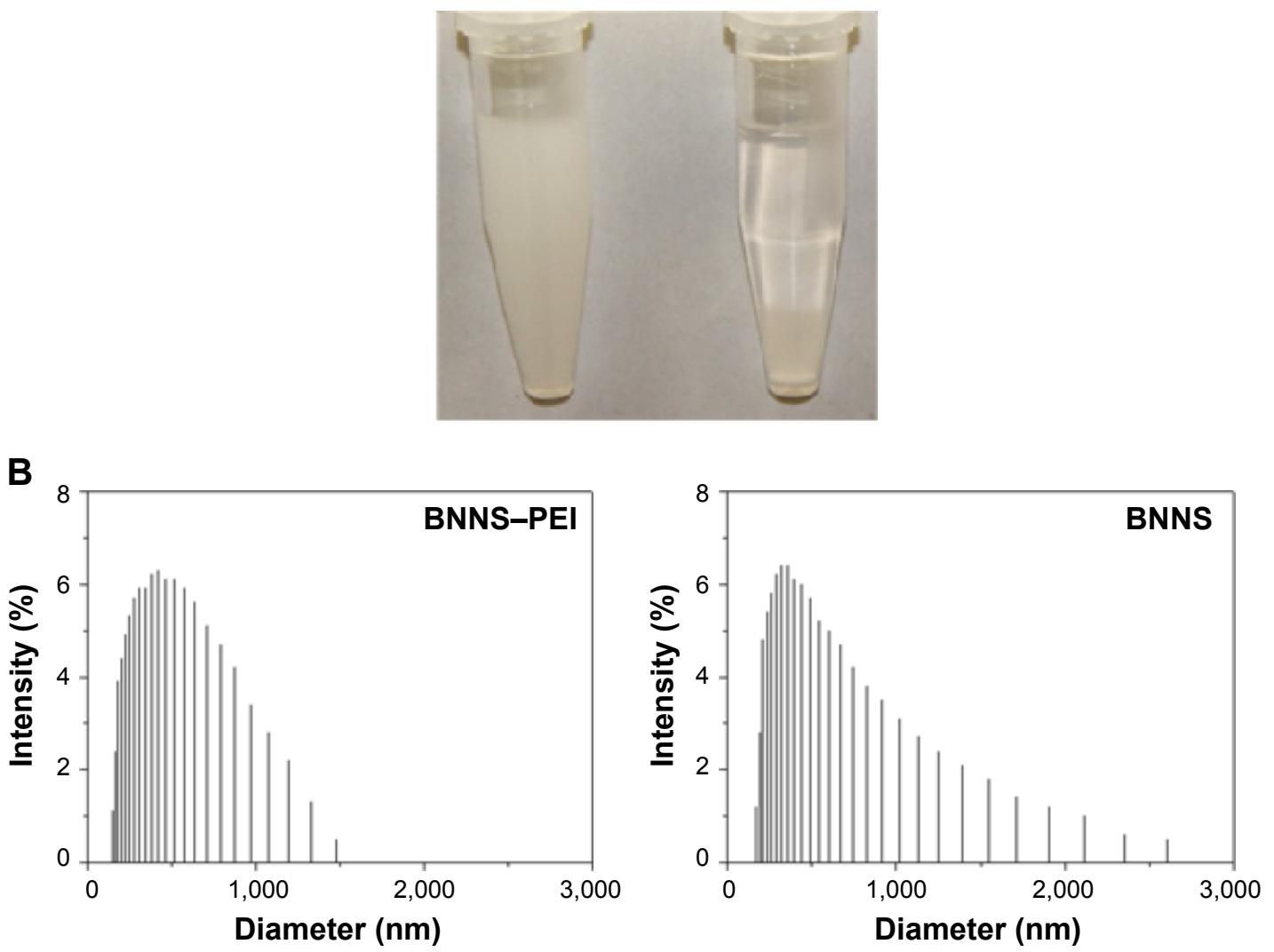

Figure 4 Characterizations of the BNNS and BNNS-PEI complexes.

Notes: (A) Dispersed BNNS and BNNS-PEI complexes in PBS after statically placed for 24 hours. (B) Hydrodynamic diameters distribution of BNNS and BNNS-PEI complexes in PBS.

Abbreviations: BNNS, boron nitride nanospheres; PBS, phosphate-buffered saline; PEl, polyethyleneimine.

mainly through the interaction of the amino functional group in PEI with BNNS. PEI coating on the surface can trigger electrostatic and steric repulsion among the highly positively charged BNNS-PEI complexes in solutions and stabilize the dispersion. Further measurement of the size distributions showed that PEI coating did not significantly increase the mean particle size of the BNNS-PEI complexes, which exhibited a relatively narrow size distribution in PBS compared with BNNS (Figure 4B). This phenomenon also indicates that the BNNS-PEI complexes may have favorable dispersity in PBS. Thus, the BNNS-PEI complexes can be used as carriers for $\mathrm{CpG}$ ODNs delivery.

\section{In vitro cytotoxicity}

The cytotoxicity of a carrier is critical for its application in drug delivery systems. ${ }^{36}$ In the present study, the in vitro cytotoxicity of the BNNS-PEI complexes was evaluated using human PBMCs in a water-soluble tetrazolium cell proliferation assay. PEI is a widely used nonviral gene delivery carrier, but it is reportedly toxic to cells. In the present study, both BNNS and BNNS-PEI complexes with concentrations of up to $100 \mu \mathrm{g} / \mathrm{mL}$ showed slight cytotoxicity to human PBMCs (Figure 5). This phenomenon may be mainly attributed to the low molecular weight (600 Da) of PEI used in our experiment. The cytotoxicity of PEI is closely dependent on its structure and molecular

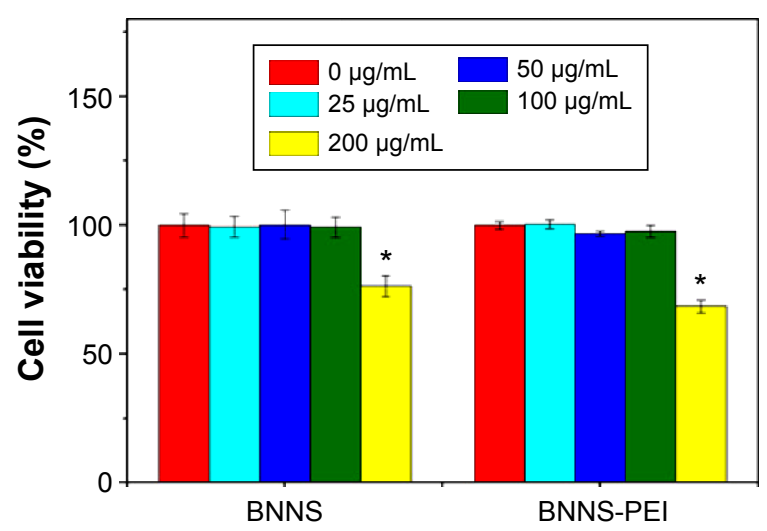

Figure 5 In vitro cytotoxicity assay. Relative cell viability of peripheral blood mononuclear cells incubated with increasing concentrations of BNNS or BNNS-PEI complexes measured by a water-soluble tetrazolium salt assay.

Notes: Data are presented as mean \pm standard deviation $(n=5) . * P<0.05$.

Abbreviations: BNNS, boron nitride nanospheres; $\mathrm{PEI}$, polyethyleneimine. 
weight. Low-molecular weight PEI always shows much lower cytotoxicity compared with high-molecular weight PEI ${ }^{37}$ However, BNNS and the BNNS-PEI complexes at $200 \mu \mathrm{g} / \mathrm{mL}$ decreased cell viability. The concentration of the BNNS-PEI complexes used in the following cell experiments was $50 \mu \mathrm{g} / \mathrm{mL}$. Therefore, PEI coating did not significantly enhance the cytotoxicity of BNNS to PBMCs, and the BNNS-PEI complexes could be used as carriers for CpG ODNs delivery.

\section{CpG ODNs loading}

The capability of the BNNS-PEI complexes as carriers for $\mathrm{CpG}$ ODNs delivery was investigated by incubating the complexes with CpG ODNs. Loading of CpG ODNs onto the BNNS-PEI complexes was initially confirmed through gel electrophoresis. The band of $\mathrm{CpG}$ ODNs from the supernatant became weaker when the BNNS-PEI complexes were mixed with $\mathrm{CpG}$ ODNs at a high weight ratio (BNNS-PEI/CpG ODNs) and disappeared at the ratio of 50 (Figure 6A). This result indicates the electrostatic binding of the negatively charged CpG ODNs to the positively charged BNNS-PEI complexes, and all CpG ODNs could be bound by the BNNS-PEI complexes at the weight ratio of 50. The characteristic adsorption peak at $260 \mathrm{~nm}$ for CpG ODNs appeared in the UV-vis spectra of the BNNS-PEI/CpG ODN complexes, which also confirmed the binding of $\mathrm{CpG}$ ODNs to the BNNS-PEI complexes (Figure 6B). Meanwhile, the loading capacity of the CpG ODNs on BNNS and the BNNS-PEI complexes increased with increasing CpG ODNs concentration and then reached a plateau. The BNNS-PEI complexes possessed eightfold higher capacity to load $\mathrm{CpG}$ ODNs (24 $\mu \mathrm{g} / \mathrm{mg}$ nanoparticles) than BNNS (Figure 6C). This result can be attributed to the strong positive surface charge of the BNNS-PEI complexes, which enhanced the loading of the $\mathrm{CpG}$ ODNs via electrostatic interactions. The release of CpG ODNs from the BNNS-PEI/CpG ODN complexes was further examined under conditions that corresponded to the physiological environment in TLR-9-localized endolysosomes. However, almost no CpG ODNs were released. This result suggests that the BNNS-PEI/CpG ODN complexes are

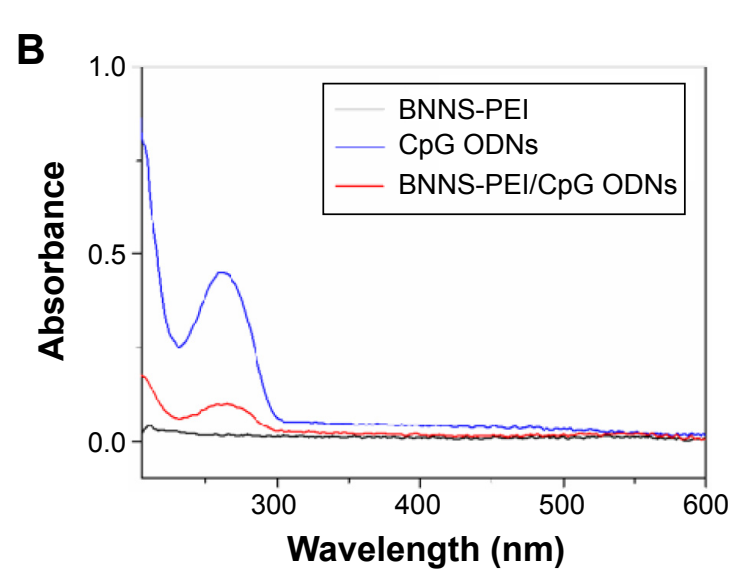

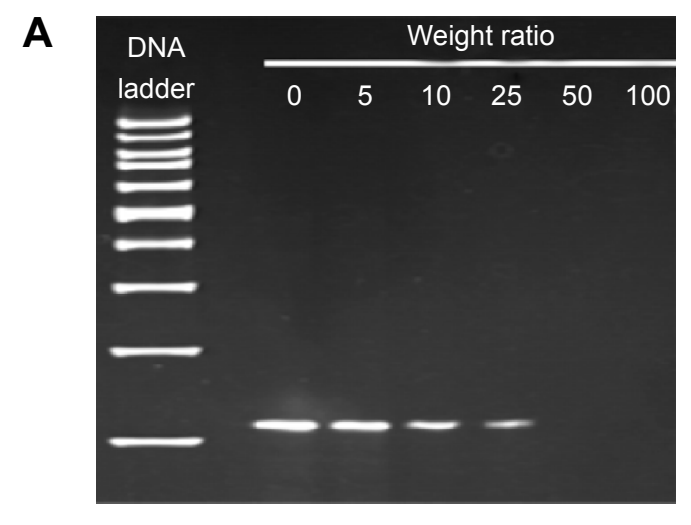

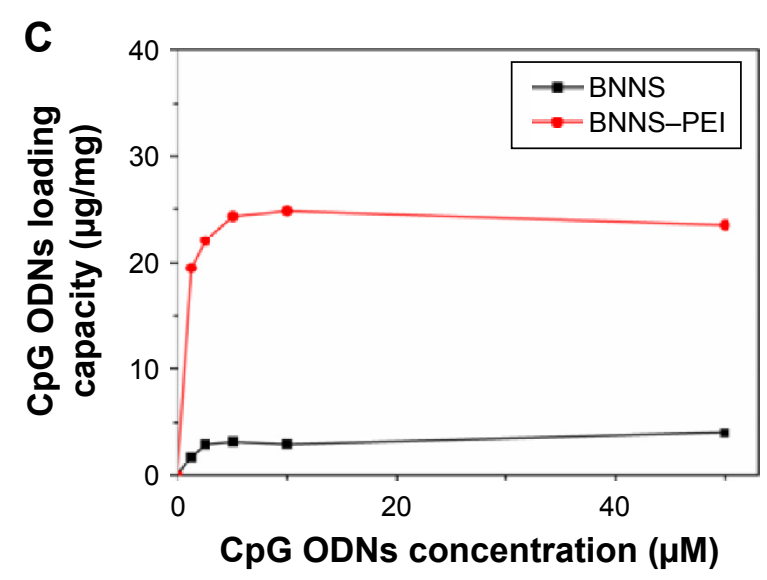

Figure 6 Loading of CPG ODNs on BNNS-PEI complexes.

Notes: (A) Gel electrophoresis image of the supernatant after interaction of CpG ODNs with BNNS-PEl at increasing weight ratios. (B) UV-vis spectra of CpG ODNs, BNNS-PEI, and BNNS-PEI/CPG ODNs complexes. (C) Loading capacity of CPG ODNs on BNNS and BNNS-PEI complexes with increasing CpG ODNs concentrations, denoted as $\mu \mathrm{g}$ C $\mathrm{pG}$ ODNs loaded on I mg BNNS. Data are presented as mean \pm standard deviation $(\mathrm{n}=3)$.

Abbreviations: BNNS, boron nitride nanospheres; ODN, oligodeoxynucleotide; PEl, polyethyleneimine. 
stable and that $\mathrm{CpG}$ ODNs cannot be easily released from the BNNS-PEI complexes because of the strong binding.

\section{Intracellular uptake of CpG ODNs}

Delivery or transfection efficiency of the carrier is a critical criterion for a drug/gene delivery system. The colloidal characteristics and CpG ODNs loading capacity of the BNNSPEI complexes may have been improved, but their capability to enhance the cellular uptake of $\mathrm{CpG}$ ODNs required further investigation. In the cellular uptake study, FITC-labeled $\mathrm{CpG}$ ODNs were used to replace the regular $\mathrm{CpG}$ ODNs and loaded on BNNS and the BNNS-PEI complexes. This process produced complexes with green fluorescence when observed via confocal laser scanning microscope. Figure 7 shows that the green fluorescence from the cells incubated with the BNNS-PEI/FITC-CpG ODN complexes was much stronger than that from the cells incubated with the BNNS/ FITC-CpG ODN complexes. However, no fluorescence was observed when free FITC-labeled CpG ODNs were applied to the cells, indicating the low cellular uptake and carrier requirement for $\mathrm{CpG}$ ODNs delivery. These findings confirmed our hypothesis that the intracellular delivery of CpG ODNs can be enhanced by the BNNS-PEI complexes. After being uptaken by cells, CpG ODNs localize mainly
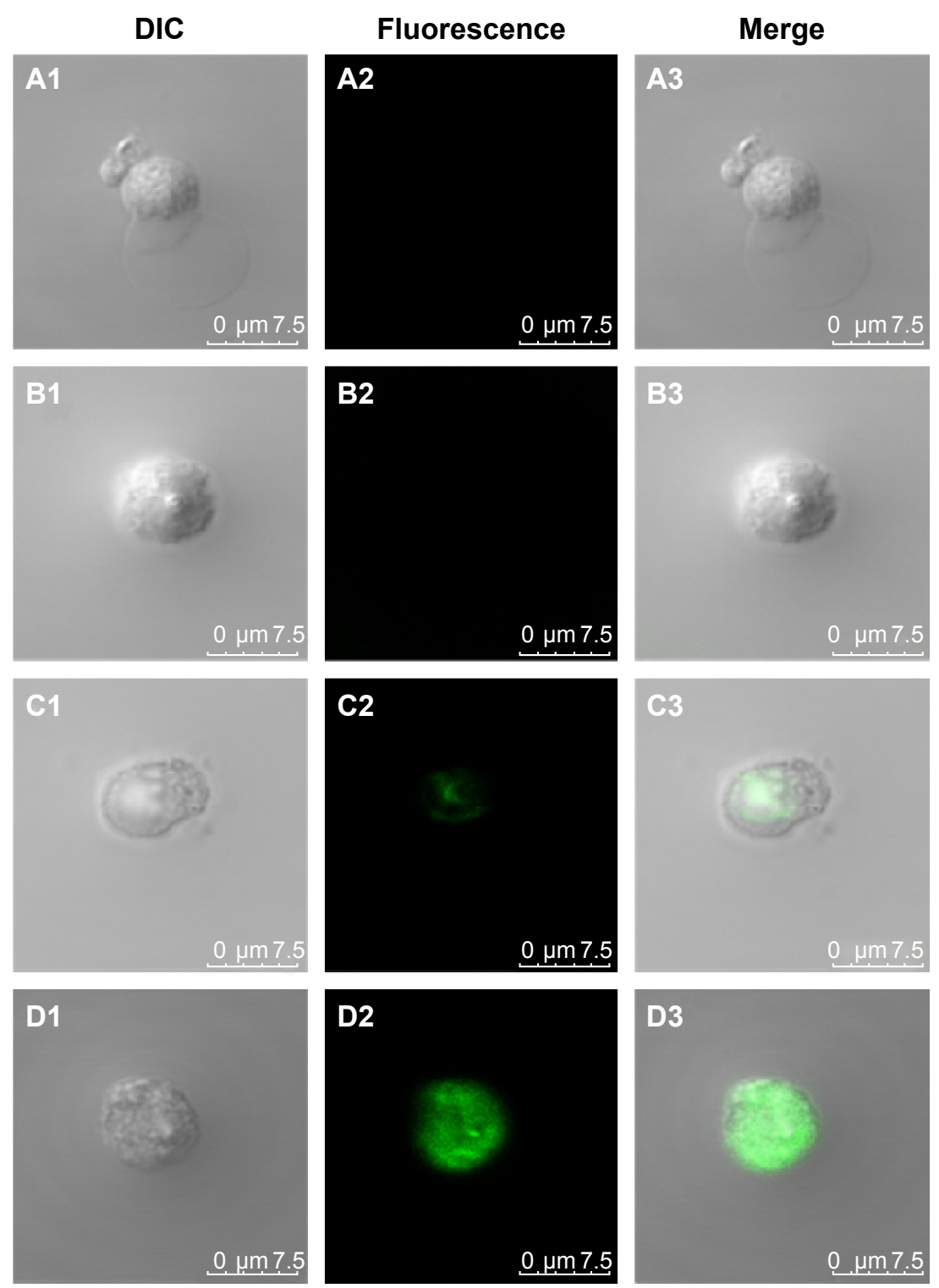

Figure 7 Confocal microscope images of PBMCs after 24 hours of incubation with BNNS (AI-3), free CpG ODNs (BI-3), CpG ODNs loaded on BNNS (CI-3), CPG ODNs loaded on BNNS-PEI complexes (DI-3).

Note: $C_{P}$ G ODNs were labeled with FITC.

Abbreviations: BNNS, boron nitride nanospheres; DIC, differential interference contrast; FITC, fluorescein isothiocyanate; ODN, oligodeoxynucleotide; PBMCs, peripheral blood mononuclear cells; PEI, polyethyleneimine. 
in endosomal compartments and activate TLR-9.,238,39 This phenomenon induces several cytokines and chemokines, as well as further modulates the immune response. ${ }^{10}$ Therefore, enhancing the cellular uptake of CpG ODNs by the BNNSPEI complexes is believed to strengthen the interaction of CpG ODNs with TLR-9 and enhance immune response.

\section{Cytokine induction in vitro}

The efficacy of the BNNS-PEI complexes as carriers for $\mathrm{CpG}$ ODNs delivery was tested by incubating BNNS-PEI/CpG ODNs with PBMCs. The resultant cytokines were determined via enzyme-linked immunosorbent assay. As expected, PBMCs stimulated by the BNNS-PEI/CpG ODN complexes had the highest amounts of IL-6 and TNF- $\alpha$ (Figure 8A, B), reaching levels approximately six times higher than those in the cells stimulated by BNNS/CpG ODNs. Although PEI can be used alone as a drug delivery carrier, the BNNS-PEI complexes exhibited much higher efficiency, and the BNNS-PEI/CpG
ODN complexes induced higher amounts of cytokines (Figure 8A, B). By contrast, free CpG ODNs can only induce a relatively small amount of cytokines. This finding suggests that the high cytokine production of $\mathrm{CpG}$ ODNs was due to their high loading capacity and enhanced cellular uptake resulting from the positively charged BNNS-PEI complexes. Similar results were obtained when a BNNS-binding peptide was used as a linker molecule for $\mathrm{CpG}$ ODNs delivery; this peptide improved the $\mathrm{CpG}$ ODNs internalization by PBMCs. ${ }^{40}$ However, the simultaneous induction of IL- 6 and IFN- $\alpha$ was not achieved with our previous BNNS delivery system. ${ }^{39,40}$

Class B CpG ODNs, which have a linear structure, can stimulate TLR-9 in B cells and induce IL-6 and TNF- $\alpha$; however, these ODNs cannot induce IFN- $\alpha$ similar to class A CpG ODNs. ${ }^{10,41-43}$ When we applied the CpG ODNs loaded electrostatically onto PEI-functionalized BNNS to stimulate the PBMCs, class B CpG ODNs acquired the capability to induce IFN- $\alpha$ (Figure 8C). By contrast, free CpG ODNs or the
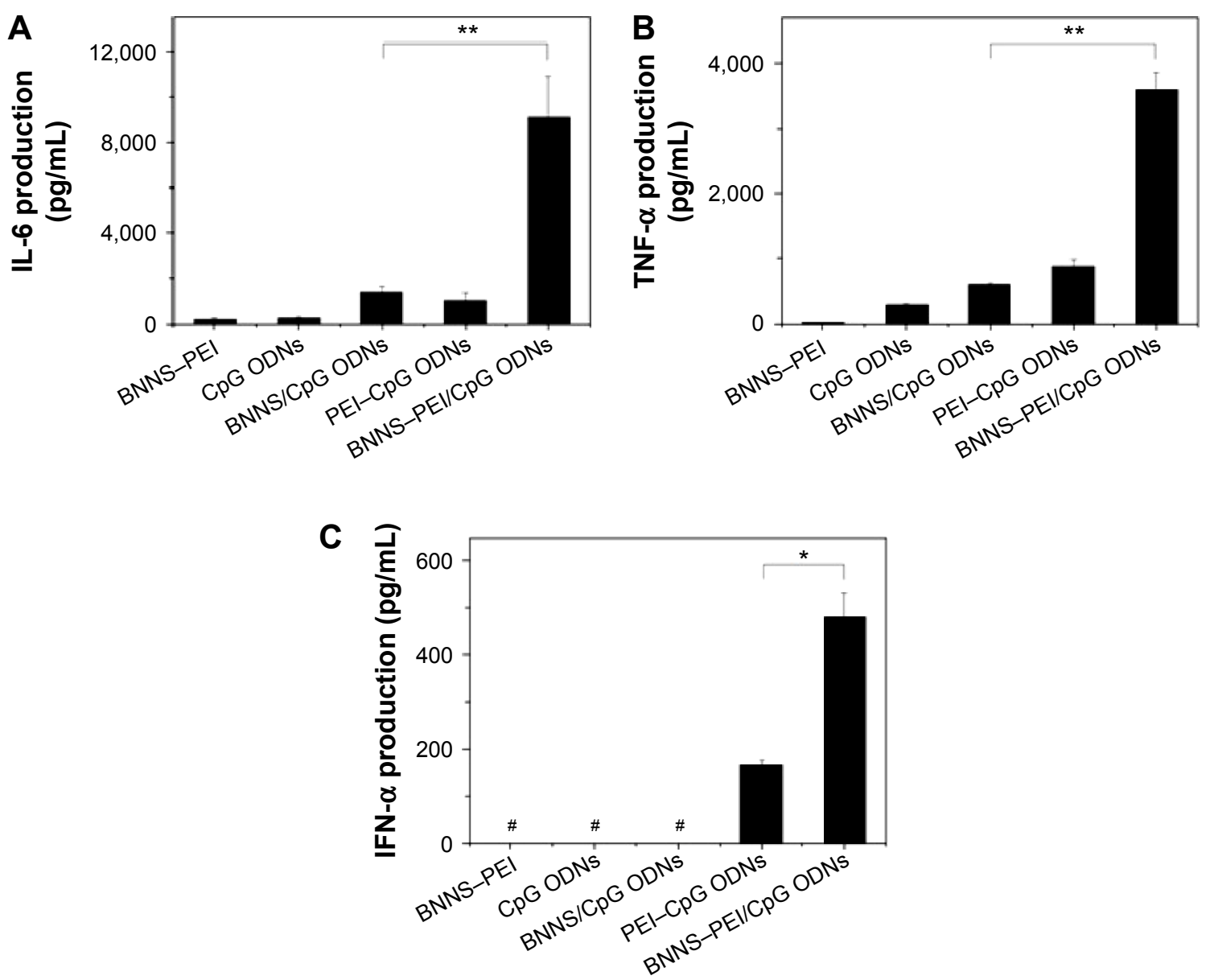

Figure 8 Cytokine induction from PBMCs stimulated by CPG ODNs loaded on BNNS and BNNS-PEl complexes.

Notes: Loaded BNNS or BNNS-PEI complexes $(50 \mu \mathrm{g} / \mathrm{mL})$. (A) IL-6 production. (B) TNF- $\alpha$ production. (C) IFN- $\alpha$ production. Data are presented as mean \pm standard deviation ( $\mathrm{n}=3)$. $* P<0.05$, $* * P<0.01$. " not detectable (below detection limit).

Abbreviations: BNNS, boron nitride nanospheres; IFN, interferon; IL-6, interleukin-6; ODN, oligodeoxynucleotide; PBMCs, peripheral blood mononuclear cells; PEI, polyethyleneimine; TNF, tumor necrosis factor. 
CpG ODNs directly loaded on BNNS cannot stimulate IFN- $\alpha$ secretion. IFN- $\alpha$ induction can also be attributed to the $\mathrm{CpG}$ ODNs loaded on the BNNS-PEI complexes but not to the free ones released from the BNNS-PEI/CpG ODN complexes. We did not detect the release of CpG ODNs from the BNNS-PEI/ CpG ODN complexes under conditions that corresponded to the physiological environment in the TLR-9-localized endolysosome. A high amount of IFN- $\alpha$ induction was also reported when class $\mathrm{B}$ CpG ODNs were adsorbed electrostatically onto polystyrene nanoparticles or silica nanoparticles with cationic polymer-coated surfaces. ${ }^{30}$ The mechanism by which class B CpG ODNs acquire the potential to induce IFN- $\alpha$ remains unclear, but the physical form of the CpG ODNs may play an important role in this process. ${ }^{18,44,45}$

The immunostimulatory effects of CpG ODNs depend not only on their base sequence and structure but also on their physical form. ${ }^{44}$ The greater capability of class A $\mathrm{CpG}$ ODNs to induce IFN- $\alpha$ production is due to the formation of a higher order multimeric structure. ${ }^{30}$ However, such structures are not observed in class B CpG ODNs, which form linear structures. ${ }^{46}$ An immune response similar to class A $\mathrm{CpG}$ ODNs can be obtained by artificially causing class $\mathrm{B} \mathrm{CpG}$ ODNs to form a multimeric structure using nanoparticles. ${ }^{33,47}$ This phenomenon suggests that the multimerization of $\mathrm{CpG}$ ODNs is crucial for IFN- $\alpha$ induction. Therefore, the class B CpG ODNs loaded onto the positively charged BNNS-PEI complexes through electrostatic interactions formed a higher order multimeric structure similar to class A CpG ODNs and acquired the capability to induce IFN- $\alpha$. However, similar to free class B CpG ODNs, class B CpG ODNs could not form a multimeric structure when bound directly to negatively charged BNNS because of the weak interactions. Thus, class B CpG ODNs can induce IL-6 and TNF- $\alpha$ but not IFN- $\alpha$. However, the exact mechanism by which class B CpG ODNs acquire the capability to induce IFN- $\alpha$ remains unclear, and further investigation is currently underway.

\section{Conclusion}

PEI-functionalized BNNS were used as nanocarriers for the intracellular delivery of CpG ODNs. PEI was coated on the surface of BNNS through noncovalent electrostatic interactions. The resultant BNNS-PEI complexes possessed strong positive charges and enhanced dispersity and stability in aqueous solution. Furthermore, the BNNS-PEI complexes were nontoxic to human PBMCs up to a concentration of $100 \mu \mathrm{g} / \mathrm{mL}$. Given their positive surface charge, the BNNS-PEI complexes can effectively enhance the loading capacity and cellular uptake of CpG ODNs. The use of the BNNS-PEI complexes as carriers for $\mathrm{CpG}$ ODNs delivery significantly enhanced the production of cytokines, such as IL-6 and TNF- $\alpha$, from PBMCs compared with the use of BNNS or PEI. Class B CpG ODNs loaded on the BNNS-PEI complexes acquired the capability to induce IFN- $\alpha$. PEI coating may have changed the physical form of class B CpG ODNs on BNNS, further modulated their interaction with TLR-9, and induced both IL-6 and IFN- $\alpha$. Our results highlight the potential of the BNNS-PEI complexes as novel nanocarriers for enhancing the immunostimulatory and therapeutic activity of $\mathrm{CpG}$ ODNs, especially for treating diseases that require IL-6, TNF- $\alpha$, and IFN- $\alpha$ simultaneously.

\section{Acknowledgments}

This work was supported by Fundamental Research Funds for the Central Universities (No JUSRP11427) and Scientific Research Foundation for the Returned Overseas Chinese Scholars, State Education Ministry.

\section{Disclosure}

The author reports no conflicts of interest in this work.

\section{References}

1. Krieg AM, Yi AK, Matson S, et al. CpG motifs in bacterial DNA trigger direct B-cell activation. Nature. 1995;374(6522):546-549.

2. Klinman DM. Immunotherapeutic uses of $\mathrm{CpG}$ oligodeoxynucleotides. Nat Rev Immunol. 2004;4(4):248-257.

3. Hemmi H, Takeuchi O, Kawai T, et al. A toll-like receptor recognizes bacterial DNA. Nature. 2000;408(6813):740-745.

4. Wagner H. Bacterial CpG DNA activates immune cells to signal infectious danger. Adv Immunol. 1999;73:329-368.

5. Akira S, Takeda K, Kaisho T. Toll-like receptors: critical proteins linking innate and acquired immunity. Nat Immunol. 2001;2(8):675-680.

6. Klinman DM, Yi AK, Beaucage SL, Conover J, Krieg AM. CpG motifs present in bacterial DNA rapidly induce lymphocytes to secrete interleukin 6, interleukin 12, and interferon gamma. Proc Natl Acad Sci U S A. 1996;93(7):2879-2883.

7. Bauer S, Kirschning CJ, Häcker H, et al. Human TLR9 confers responsiveness to bacterial DNA via species-specific $\mathrm{CpG}$ motif recognition. Proc Natl Acad Sci U S A. 2001;98(16):9237-9242.

8. Stacey KJ, Sweet MJ, Hume DA. Macrophages ingest and are activated by bacterial DNA. J Immunol. 1996;157(5):2116-2122.

9. Sun SQ, Zhang XH, Tough DF, Sprent J. Type I interferon-mediated stimulation of T cells by CgG DNA. J Exp Med. 1998;188(12):2335-2342.

10. Krieg AM. CpG motifs in bacterial DNA and their immune effects. Annu Rev Immunol. 2002;20:709-760.

11. Fonseca DE, Kline JN. Use of CpG oligonucleotides in treatment of asthma and allergic disease. Adv Drug Deliv Rev. 2009;61(3):256-262.

12. Klinman DM, Klaschik S, Sato T, Tross D. CpG oligonucleotides as adjuvants for vaccines targeting infectious diseases. Adv Drug Deliv Rev. 2009;61(3):248-255.

13. Krieg AM. Therapeutic potential of toll-like receptor 9 activation. Nat Rev Drug Discov. 2006;5(6):471-484.

14. Murad YM, Clay TM. CpG oligodeoxynucleotides as TLR9 agonists therapeutic applications in cancer. Bio Drugs. 2009;23(6):361-375.

15. Klinman DM, Currie D, Gursel I, Verthelyi D. Use of CpG oligodeoxynucleotides as immune adjuvants. Immunol Rev. 2004;199(1):201-216.

16. Mutwiri G, Littel-van Den Hurk SV, Babiuk LA. Approaches to enhancing immune responses stimulated by $\mathrm{CpG}$ oligodeoxynucleotides. $\mathrm{Adv}$ Drug Deliv Rev. 2009;61(3):226-232. 
17. Mutwiri GK, Nichani AK, Babiuk S, Babiuk LA. Strategies for enhancing the immunostimulatory effects of $\mathrm{CpG}$ oligodeoxynucleotides. J Control Release. 2004;97(1):1-17.

18. Hanagata N. Structure-dependent immunostimulatory effect of $\mathrm{CpG}$ oligodeoxynucleotides and their delivery system. Int J Nanomedicine. 2012; 7:2181-2195.

19. Sheehan JP, Lan HC. Phosphorothioate oligonucleotides inhibit the intrinsic tenase complex. Blood. 1998;92(5):1617-1625.

20. Henry SP, Beattie G, Yeh G, et al. Complement activation is responsible for acute toxicities in rhesus monkeys treated with a phosphorothioate oligodeoxynucleotide. Int Immunopharmacol. 2002;2(12): 1657-1666.

21. Chopra NG, Luyken RJ, Cherrey K, et al. Boron-nitride nanotubes. Science. 1995;269(5226):966-967.

22. Golberg D, Bando Y, Tang CC, Zhi CY. Boron nitride nanotubes. Adv Mater. 2007;19(18):2413-2432.

23. Chen X, Wu P, Rousseas M, et al. Boron nitride nanotubes are noncytotoxic and can be functionalized for interaction with proteins and cells. J Am Chem Soc. 2009;131(3):890-891.

24. Jonsson E, Simonsen L, Karlsson M, Larsson R. The hollow fiber model: a new method for in vivo-evaluation of antitumor effect, toxicity and pharmacokinetics of new anticancer drugs. Ann Oncol. 1998;9: 44-44.

25. Gao ZH, Zhi CY, Bando Y, Golberg D, Serizawa T. Isolation of individual boron nitride nanotubes via peptide wrapping. J Am Chem Soc. 2010; 132(14):4976-4977.

26. Ciofani G, Raffa V, Menciassi A, Cuschieri A. Boron nitride nanotubes an innovative tool for nanomedicine. Nano Today. 2009;4(1):8-10.

27. Ciofani G, Danti S, Nitti S, Mazzolai B, Mattoli V, Giorgi M. Biocompatibility of boron nitride nanotubes: an up-date of in vivo toxicological investigation. Int J Pharm. 2013;444(1-2):85-88.

28. Ciofani G, Danti S, Genchi GG, et al. Pilot in vivo toxicological investigation of boron nitride nanotubes. Int J Nanomedicine. 2012;7: 19-24.

29. Zhi CY, Meng WJ, Yamazaki T, et al. BN nanospheres as CpG ODN carriers for activation of toll-like receptor 9. J Mater Chem. 2011;21(14): 5219-5222.

30. Kerkmann M, Costa LT, Richter C, et al. Spontaneous formation of nucleic acid-based nanoparticles is responsible for high interferon- $\alpha$ induction by CpG-A in plasmacytoid dendritic cells. J Biol Chem. 2005; 280(9):8086-8093.

31. Putnam D, Gentry CA, Pack DW, Langer R. Polymer-based gene delivery with low cytotoxicity by a unique balance of side-chain termini. Proc Natl Acad Sci US A. 2001;98(3):1200-1205.

32. Zhu J, Tang A, Law LP, et al. Amphiphilic core-shell nanoparticles with poly(ethylenimine) shells as potential gene delivery carriers. Bioconjug Chem. 2005;16(1):139-146.

33. Manoharan Y, Ji Q, Yamazaki T, et al. Effect of molecular weight of polyethyleneimine on loading of $\mathrm{CpG}$ oligodeoxynucleotides onto flake-shell silica nanoparticles for enhanced TLR9-mediated induction of interferon-alpha. Int J Nanomedicine. 2012;7:3625-3635.
34. Meng WJ, Yamazaki T, Nishida Y, Hanagata N. Nuclease-resistant immunostimulatory phosphodiester $\mathrm{CpG}$ oligodeoxynucleotides as human toll-like receptor 9 agonists. BMC Biotechnol. 2011;11:88.

35. Tang CC, Bando Y, Huang Y, Zhi CY, Golberg D. Synthetic routes and formation mechanisms of spherical boron nitride nanoparticles. Adv Funct Mater. 2008;18(22):3653-3661.

36. Zhu YF, Ikoma T, Hanagata N, Kaskel S. Rattle-type $\left.\left.\mathrm{Fe}_{3}\right) \mathrm{O}_{4}\right) @$ $\mathrm{SiO}\left({ }_{2}\right)$ hollow mesoporous spheres as carriers for drug delivery. Small. 2010;6(3):471-478.

37. Brunot C, Ponsonnet L, Lagneau C, Farge P, Picart C, Grosgogeat B. Cytotoxicity of polyethyleneimine (PEI), precursor base layer of polyelectrolyte multilayer films. Biomaterials. 2007;28(4):632-640.

38. Latz E, Schoenemeyer A, Visintin A, et al. TLR9 signals after translocating from the ER to CpG DNA in the lysosome. Nat Immunol. 2004;5(2): 190-198.

39. Zhang H, Chen S, Zhi C, Yamazaki T, Hanagata N. Chitosan-coated boron nitride nanospheres enhance delivery of $\mathrm{CpG}$ oligodeoxynucleotides and induction of cytokines. Int J Nanomedicine. 2013;8: 1783-1793.

40. Zhang H, Yamazaki T, Zhi C, Hanagata N. Identification of a boron nitride nanosphere-binding peptide for the intracellular delivery of $\mathrm{CpG}$ oligodeoxynucleotides. Nanoscale. 2012;4(20):6343-6350.

41. Krug A, Towarowski A, Britsch S, et al. Toll-like receptor expression reveals CpG DNA as a unique microbial stimulus for plasmacytoid dendritic cells which synergizes with CD40 ligand to induce high amounts of IL-12. Eur J Immunol. 2001;31(10):3026-3037.

42. Hartmann G, Weeratna RD, Ballas ZK, et al. Delineation of a CpG phosphorothioate oligodeoxynucleotide for activating primate immune responses in vitro and in vivo. J Immunol. 2000;164(3):1617-1624.

43. Hartmann G, Krieg AM. Mechanism and function of a newly identified CpG DNA moth in human primary B cells. J Immunol. 2000;164(2): 944-952.

44. Guiducci C, Ott G, Chan JH, et al. Properties regulating the nature of the plasmacytoid dendritic cell response to toll-like receptor 9 activation. J Exp Med. 2006;203(8):1999-2008.

45. Honda K, Yanai H, Negishi H, et al. IRF-7 is the master regulator of type-I interferon-dependent immune responses. Nature. 2005;434(7034): $772-777$.

46. Klein DCG, Latz E, Espevik T, Stokke BT. Higher order structure of short immunostimulatory oligonucleotides studied by atomic force microscopy. Ultramicroscopy. 2010;110(6):689-693.

47. Chinnathambi S, Chen S, Ganesan S, Hanagata N. Binding mode of CpG oligodeoxynucleotides to nanoparticles regulates bifurcated cytokine induction via toll-like receptor 9. Sci Rep. 2012;2:534.
International Journal of Nanomedicine

\section{Publish your work in this journal}

The International Journal of Nanomedicine is an international, peerreviewed journal focusing on the application of nanotechnology in diagnostics, therapeutics, and drug delivery systems throughout the biomedical field. This journal is indexed on PubMed Central, MedLine, CAS, SciSearch $®$, Current Contents $\AA /$ Clinical Medicine,

\section{Dovepress}

Journal Citation Reports/Science Edition, EMBase, Scopus and the Elsevier Bibliographic databases. The manuscript management system is completely online and includes a very quick and fair peer-review system, which is all easy to use. Visit http://www.dovepress.com/ testimonials.php to read real quotes from published authors. 